\title{
¿Qué quieren saber los usuarios de éxtasis?: estudio de una lista de correo de Internet
}

\author{
Fernando Caudevilla Gálligo \\ Instituto Madrileño del Menor y la Familia (IMMF). Comunidad Autónoma de Madrid. \\ Enviar correspondencia a: \\ Fernando Caudevilla Gálligo. C/ López de Hoyos, 133 Esc 1, 2 1. 28002 Madrid. Tlf. 609914 409. e-mail: caudevilla@hotmail.com
}

Recibido: 27 de febrero de 2004 Aceptado: 22 de septiembre de 2004

\section{RESUMEN}

Objetivo: Obtener información acerca de las creencias, actitudes y opiniones que los usuarios de éxtasis (3,4-metilendioximetanfetamina o MDMA) tienen sobre esta droga.

Material y métodos: Entre el 7 de Agosto y el 6 de Diciembre de 2003 se revisaron los 15.117 mensajes de correo electrónico enviados a una lista de correo de Internet en castellano sobre reducción de riesgos en el consumo de drogas. Se clasificaron y analizaron 681 preguntas relacionadas con el éxtasis y la salud

Resultados y conclusiones: Los mensajes con preguntas sobre las diferentes presentaciones de la MDMA constituyeron la categoría más frecuente $(n=171 ; 25.11 \%)$. Además se encontró una gran cantidad y variedad de preguntas en relación con distintos aspectos como combinaciones con fármacos ( $n=162$; $23.9 \%$ ), efectos adversos ( $n=98 ; 14.39 \%$ ), combinaciones con drogas $(n=69 ; 10.13 \%)$ o toxicidad $(n=61 ; 8.96 \%)$ entre otros. Algunos de los resultados obtenidos son congruentes con los de estudios previos. La repetición de determinadas cuestiones que no han sido descritas previamente en la literatura científica (posología, fármacos con efecto neuroprotector, efectos de pastillas concretas...) sugiere que foros como el estudiado pueden constituir una importante fuente de información sobre el patrón de consumo de nuevas drogas así como una herramienta preventiva complementaria.

Palabras clave: MDMA, éxtasis, Internet, drogas de síntesis, prevención.

\section{ABSTRACT}

Objective: To obtain information on beliefs, attitudes and opinions that ecstasy (3,4-3,4-methylenedioxymethamphetam ine or MDMA) users have about this drug.

Material and methods: 15,117 e-mails sent to a Spanish Internet mailing list dealing with risk reduction in drug use were checked between 6 August and 7 December 2003. 681 questions related to ecstasy and health were classified and analyzed.

Results and conclusions: e-mails containing questions about the different presentation of MDMA were the most commonly found ( $n=171 ; 25.11 \%)$. In addition, there was a large number and variety of questions related to other aspects such as its combination with prescription drugs $(n=162 ; 23.9 \%)$, adverse effects ( $n=98 ; 14.39 \%)$, combination with illegal drugs $(n=69$; $10.13 \%)$ or toxicity $(n=61 ; 8.96 \%)$. Some of the results that were obtained are consistent with those from previous studies. The repetition of specific questions, not previously described in scientific bibliography (dosage, neuroprotective drugs, effects of specific pills ...), suggests that mailing lists like the one reviewed here can become an important source of information about drug use habits, as well as a complementary preventive tool.

Key Words: MDMA, ecstasy, Internet, designer drugs, prevention.

\section{1.- INTRODUCCIÓN}

$\mathbf{L}$ a expansión del uso de nuevas drogas, la aparición de nuevos patrones de consumo asociados y sus repercusiones sociales obligan a una renovación continua de los planteamientos preventivos y terapéuticos para adecuarlos a la realidad sanitaria y social existente en cada momento. Durante la pasada década hemos asistido a un crecimiento en el consumo de las drogas de síntesis, particularmente del éxtasis (3,4-metilendioximetanfetamina o MDMA) como droga asociada a espacios recreativos, vinculándose a ciertas manifestaciones de la cultura de la música electrónica (clubs, fiestas rave, after-hours).

Un 4,2\% de la población española de 15 y 65 años afirma haber probado esta droga, porcentaje que se 
duplica entre los de 15 a 24 años (8\%) $)^{1}$ Dentro de la población escolarizada entre 14 y 18 años, un 4,1\% refiere haber consumido éxtasis en el último año y un $1,7 \%$ en el último mes ${ }^{2}$. Otros datos, como el incremento en las incautaciones policiales de MDMA, que se han multiplicado por 200 en la última década superando el millón de comprimidos en el 2002, dan idea de la importancia del fenómeno.

La investigación científica sobre la MDMA ha experimentado también un rápido crecimiento. En los últimos quince años se han publicado más de mil quinientos artículos científicos que han estudiado esta sustancia desde muy diferentes enfoques: biología molecular, estudios in vitro, ensayos en animales, administración a voluntarios, encuestas y estudios en consumidores, comunicación de casos clínicos... Los efectos neurotóxicos de la MDMA y su posible extrapolación a los patrones de consumo en humanos han recibido gran parte de la atención, siendo en la actualidad objeto de controversia entre los investigadores ${ }^{3-6}$.

Uno de los campos de investigación insuficientemente explorados hasta el momento es el estudio de las creencias, actitudes y opiniones que los consumidores de MDMA tienen sobre esta sustancia. Este tipo de estudios puede facilitar un diseño efectivo de programas preventivos destinados a los propios usuarios pero su abordaje presenta ciertas dificultades. Los consumidores son en su mayoría jóvenes sanos, que utilizan los servicios sanitarios con poca frecuencia y que no usan éstos para consultar sus problemas y dudas al consumir drogas ilegales. Además muchos profesionales de Atención Primaria no poseen una formación específica sobre el éxtasis y otras drogas de síntesis lo que dificulta una búsqueda activa de estas cuestiones. Así, la investigación de creencias y actitudes acerca de la MDMA a través de métodos tradicionales puede resultar insuficiente, lo que hace conveniente buscar estrategias complementarias.

La difusión de Internet supone una nueva herramienta que permite la transmisión de todo tipo de conocimientos de una forma rápida, igualitaria y universal. La cantidad de información disponible acerca de las drogas, en particular sobre aquellas de uso recreativo es ingente. Las relaciones entre drogas, jóvenes e Internet han comenzado a ser valoradas recientemente en la literatura científica. Los artículos publicados hasta el momento han destacado la importancia de las nuevas tecnologías como fuente de información sobre drogas ${ }^{7}$ evaluado la calidad de la información disponible en páginas web y señalando los riesgos de su uso inadecuado ${ }^{8-13}$.

Además de las páginas web existen otras aplicaciones en Internet cuyo análisis puede aportar información útil sobre lo que los consumidores de MDMA piensan sobre esta sustancia. Los foros de discusión y listas de correo son programas que permiten a un grupo de personas mantenerse en contacto e intercambiar información entre sí a través del correo electrónico. Cada vez que un miembro envía un mensaje a la lista de correo, éste es recibido por el resto de participantes, quienes a su vez pueden enviar respuestas que serán recibidas por toda la comunidad. Así, a través de Internet es posible establecer discusiones acerca de cualquier tema, aunque los participantes estén físicamente separados, de una manera inmediata y garantizando el anonimato. Estudiar los contenidos en detalle de los foros sobre MDMA puede ofrecer una perspectiva complementaria al estudio de esta droga.

\section{2.- MATERIALY MÉTODOS}

Objetivo: Conocer las preguntas que los usuarios de MDMA plantean acerca del uso de esta droga y sus efectos sobre la salud en un foro de Internet sobre drogas en castellano, a través de la cuantificación y clasificación de los mensajes enviados al foro.

Diseño: Estudio descriptivo retrospectivo cuantitativo.

Material: El foro de debate de la ONG Energy Control es una lista de correo de Internet en castellano, creada el 15 de Mayo del 2000 cuya temática es la disminución de riesgos en el consumo de drogas. Su dirección es http://es.groups.yahoo.com/group/energycontrol/join. El grupo se define como una "comunidad virtual que discute y habla sobre drogas, con el propósito de poner en común información útil para los consumidores de sustancias psicoactivas y reducir así los posibles riesgos que comporta su consumo". El número de miembros suscritos a la lista oscila entre 1100 y 1200. Ya que es posible darse de alta o de baja en la lista la cantidad total de personas que ha participado alguna vez en sus más de tres años de funcionamiento es sin duda mayor.

La elección de este foro entre los más populares en castellano en relación con las drogas (por ejemplo: http://www.psiconautica.com, http://forofad.fad.es/, http://www.lasdrogas.info/foro, http://www.ailaket.org, http://www.psiconautica.org, http://www.institutobitacora.org, http://www.cannabiscafe.net) se fundamentó en los siguientes argumentos: actividad continua y creciente desde su formación, elevado número de participantes y mensajes, persistencia en el tiempo, acceso público, posibilidad de analizar todos los mensajes enviados de forma retrospectiva y temática centrada en la reducción de riesgos y las drogas de síntesis. Aunque otros foros en inglés comparten estas características como http://www.bluelight.nu o alt.drugs.ecstasy (disponible a través de http://groups. 
google.es), se prefirió analizar el foro de Energy Control por acercarse más a la realidad de nuestro país.

Procedimiento: Entre el 15 de Mayo de 2000 y el 6 de Diciembre de 2003 se enviaron 15.117 mensajes a la lista de correo de Energy Control. Mensualmente la lista recibió una media de 340 mensajes al mes ( $n=$ 44 meses, $\sigma=206,82$ mensajes). Los mensajes fueron revisados de forma secuencial y consecutiva entre el 7 de Agosto y el 6 de Diciembre de 2003. Se registraron aquellos mensajes en los que un participante expone al resto de la comunidad alguna pregunta concreta sobre la MDMA y cuyo contenido esté relacionado con la salud: efectos, forma de presentación, dosificación, vía de administración, efectos adversos, toxicidad y riesgos, contraindicaciones e interacciones con fármacos y/o drogas.

Se establecieron nueve categorías en las que fueron clasificados los mensajes de acuerdo con su contenido. En la categoría "Presentación" se recogieron mensajes en los que se formularon preguntas relacionadas con las distintas formas concretas en la que la MDMA puede encontrarse en el mercado (comprimidos, cristal, polvo...). En las categorías "Combinaciones con fármacos" y "Combinaciones con drogas" se incluyeron las preguntas acerca de los efectos y/o riesgos de la combinación de un determinado fármaco de prescripción o droga de abuso con la MDMA. Las categorías "Efectos adversos" y "Toxicidad" recogieron aquellas preguntas acerca de los efectos adversos (entendidos como aquellos frecuentes, de carácter generalmente leve y que suelen presentarse con dosis habituales) y tóxicos (aquellos más graves, infrecuentes o que aparecen ante dosis y pautas de consumo elevadas). La categoría “Dosificación" recogió los mensajes en los que se hacen preguntas en relación con la cantidad de MDMA que permite un consumo de menor riesgo: dosis tóxica, frecuencia de consumo, periodos de descanso, máxima cantidad recomendable a consumir en una ocasión... En “Vía de administración" se recogieron cuestiones acerca de otras formas de administrar la MDMA distintas de la vía oral. Aquellas preguntas en relación con los riesgos de consumir MDMA mientras se padece alguna enfermedad concurrente 0 ante determinadas circunstancias fisiológicas (menstruación, embarazo, lactancia...) se agruparon en "Contraindicaciones médicas". Las preguntas sobre la posibilidad de detectar MDMA en fluidos orgánicos mediante analíticas de sangre y orina se agruparon en "Duración y detección en fluidos".

Se excluyeron aquellos mensajes en los que se relataban experiencias u opiniones acerca de la MDMA pero en los que no se planteaban cuestión alguna. Asimismo se excluyeron todas las respuestas a las preguntas formuladas y aquellos mensajes enviados más de una vez por error. Las cuestiones de tipo legal o jurídico tampoco fueron incluidas. Se contabilizaron como datos diferentes aquellos mensajes de correo electrónico en cuyo contenido se hacía más de una pregunta.

\section{3.-RESULTADOS}

Se registraron 681 mensajes sobre la MDMA que cumplían los criterios de inclusión para el estudio. Además, durante el proceso de revisión de los mensajes se encontraron preguntas concretas en relación con 48 sustancias distintas: cocaína $(n=81)$, metanfetamina $(n=59), \operatorname{LSD}(n=56)$, ketamina $(n=47)$, cannabis $(n=46)$, así como una amplia variedad de feniletilaminas, triptaminas de síntesis y alucinógenos de origen vegetal sumando un total de 1278 mensajes.

Los 681 mensajes sobre MDMA fueron clasificadas de acuerdo a su contenido dentro de las categorías ya definidas (Tabla 1).Los mensajes con preguntas sobre las diferentes presentaciones de la MDMA constituyeron la categoría más frecuente. Dentro de esta, la pregunta más habitual consiste en demandar información acerca de los efectos que puede producir una pastilla de un determinado logotipo $(n=101$, $14,83 \%$ del total). Además, se enviaron mensajes preguntando acerca de la composición y adulterantes de las pastillas $(n=32)$ así como sobre su caducidad $(n=7)$. Se encontraron también preguntas sobre los efectos que pueden producir otras formas de presentación de la MDMA distintas a las pastillas, como el cristal $(n=21)$ o el polvo $(n=10)$.

\section{TABLA 1: PREGUNTAS SOBRE MDMA}

\begin{tabular}{lrc}
\hline Categoría & $\mathbf{n}$ & $\%$ \\
\hline Presentación de la MDMA & 171 & $25,11 \%$ \\
Combinaciones con fármacos & 162 & $23,79 \%$ \\
Efectos adversos & 98 & $14,39 \%$ \\
Combinaciones con drogas & 69 & $10,13 \%$ \\
Toxicidad & 61 & $8,96 \%$ \\
Dosificación & 55 & $8,08 \%$ \\
Vias de administración & 32 & $4,70 \%$ \\
Contraindicaciones médicas & 20 & $2,94 \%$ \\
Duración y detección en fluidos & 13 & $1,91 \%$
\end{tabular}

Las preguntas acerca de la combinación de MDMA y fármacos se resumen en la Tabla 2. En las preguntas acerca del triptófano, antioxidantes, neuroprotectores (vitamina C, vitamina E, beta-caroteno, ácido lipoico, selenio...) e inhibidores selectivos de la recaptación de serotonina (ISRS) los miembros del foro demandan información sobre cómo utilizar estos fármacos para reducir efectos adversos o tóxicos de 


\section{TABLA 2: COMBINACIONES CON FARMACOS}

\begin{tabular}{lrr}
\hline Fármaco & $\mathbf{n}$ & \multicolumn{1}{c}{$\%$} \\
\hline Triptófano & 63 & $38,89 \%$ \\
Antioxidantes-neuroprotectores & 23 & $14,20 \%$ \\
ISRSs & 19 & $11,73 \%$ \\
IMAOs & 9 & $5,56 \%$ \\
Antihistamínicos & 6 & $3,70 \%$ \\
Anticonceptivos orales & 5 & $3,09 \%$ \\
Sildelnafilo & 5 & $3,09 \%$ \\
AlNEs & 5 & $3,09 \%$ \\
Benzodiacepinas & 4 & $2,47 \%$ \\
Paracetamol & 3 & $1,85 \%$ \\
Salbutamol & 3 & $1,85 \%$ \\
Antirretrovirales & 2 & $1,23 \%$ \\
Isotretinoína & 2 & $1,23 \%$ \\
Hormonas tiroideas & 2 & $1,23 \%$ \\
Otros & 11 & $6,82 \%$ \\
\end{tabular}

la MDMA; en el resto se pregunta sobre los riesgos específicos que pueden aparecer al utilizar de forma simultánea un determinado fármaco de prescripción y la MDMA. Con respecto a los efectos adversos (Tabla 3 ) se observó que en un gran número de ocasiones $(n=43)$ las preguntas parten de una experiencia del consumidor en la que han aparecido efectos adversos y se desea conocer si éstos pueden ser atribuibles a la sustancia.

\section{TABLA 3: EFECTOS ADVERSOS}

\begin{tabular}{lcr}
\hline Efecto adverso & $\mathbf{n}$ & $\%$ \\
\hline Psicológicos & 16 & $16,33 \%$ \\
Alucinaciones & 13 & $13,27 \%$ \\
Digestivos & 12 & $12,24 \%$ \\
Trismos & 8 & $8,16 \%$ \\
Mareo & 7 & $7,14 \%$ \\
Diplopia-nistagmus & 6 & $6,12 \%$ \\
Mialgias & 5 & $5,10 \%$ \\
Impotencia & 5 & $5,10 \%$ \\
Dificultad para orinar & 4 & $4,08 \%$ \\
Taquicardias & 4 & $4,08 \%$ \\
Cefalea & 4 & $4,08 \%$ \\
Diarrea & 3 & $3,06 \%$ \\
Parestesias & 3 & $3,06 \%$ \\
Otros & 8 & $8,16 \%$ \\
\hline
\end{tabular}

En los mensajes sobre combinaciones con drogas (Tabla 4) se encontraron preguntas sobre efectos esperables al mezclar dos drogas $(n=42)$ así como los riesgos asociados a una combinación $(n=40)$. Dentro de esta categoría se consideraron "estimulantes" la cocaína $(n=10)$ y la metanfetamina $(n=9)$ y "otras feniletilaminas de síntesis" la 4-bromo-2,5-dimetoxifeniletilamina (2C-B) $(n=3), 3,4$-metilendioxietilanfetamina

\section{TABLA 4: COMBINACIONES CON DROGAS}

\begin{tabular}{lrc}
\hline Droga & $\mathbf{n}$ & $\%$ \\
\hline Estimulantes & 19 & $27,54 \%$ \\
Alcohol & 12 & $17,39 \%$ \\
LSD & 7 & $10,14 \%$ \\
Cannabis & 7 & $10,14 \%$ \\
Otras feniletilaminas de sintesis & 7 & $10,14 \%$ \\
GHB & 5 & $7,25 \%$ \\
Mescalina & 4 & $5,80 \%$ \\
Psilocibina & 3 & $4,35 \%$ \\
Ketamina & 3 & $4,35 \%$ \\
DXM & 1 & $1,45 \%$ \\
Extasis herbal (efedrina) & 1 & $1,45 \%$ \\
\hline
\end{tabular}

(MDEA) ( $n=2$ ), 4-iodo-2,5-dimetoxifeniletilamina (2CI) $(n=1)$ y 4 -propiltio-2,5dimetoxifeniletilamina (2C-T-7) $(n=1)$.

Los resultados de las preguntas sobre toxicidad se recogen en la tabla 5. Dentro de la categoría "Dosificación" las preguntas en relación con la tolerancia farmacológica $(n=14)$ y frecuencia de consumo recomendable $(n=8)$ fueron las más frecuentes. Los resultados sobre vías de administración se recogen en la tabla 6. Las contraindicaciones médicas más consultadas fueron el asma $(n=3)$, menstruación $(n=3)$, cirugía $(n=2)$ además de una larga lista de enfermedades objeto de consulta en una única ocasión (hipotiroidismo, epilepsia, embarazo, ulcus péptico,...). En 13 ocasiones se preguntó acerca del tiempo en el que la MDMA puede detectarse en fluidos biológicos.

\begin{tabular}{|c|c|c|}
\hline \multicolumn{3}{|c|}{ TABLA 5: TOXICIDAD } \\
\hline Efecto & n & $\%$ \\
\hline Neurotoxicidad & 39 & $63,93 \%$ \\
\hline Hiponatremia & 8 & $13,11 \%$ \\
\hline Psiquiátrica & 7 & $11,48 \%$ \\
\hline Hepatotoxicidad & 5 & $8,20 \%$ \\
\hline Hipertermia & 1 & $1,64 \%$ \\
\hline Hipertension & 1 & $1,64 \%$ \\
\hline \multicolumn{3}{|c|}{ TABLA 6: VIA DE ADMINISTRACION } \\
\hline Vía & n & $\%$ \\
\hline Rectal & 9 & $28,13 \%$ \\
\hline Intravenoso & 7 & $21,88 \%$ \\
\hline Intranasal & 7 & $21,88 \%$ \\
\hline Sublingual & 6 & $18,75 \%$ \\
\hline Fumado & 3 & $9,38 \%$ \\
\hline
\end{tabular}




\section{4.- DISCUSIÓN}

Los programas preventivos destinados a consumidores de drogas deben partir de información concreta, detallada y actualizada sobre los patrones de consumo y formas de uso de las sustancias. En el caso de la MDMA y otras drogas de síntesis nos encontramos ante sustancias relativamente nuevas que producen escasa repercusión en los dispositivos sanitarios (tanto en Atención Primaria como en centros específicos para problemas de drogas). Así, la obtención de información válida y fiable que proceda de la experiencia directa de los consumidores puede ser difícil; el uso de métodos indirectos como el análisis de datos procedentes de Internet puede constituir una herramienta de trabajo complementaria a otros métodos de investigación. Sin embargo, las revisiones de listas de correo de Internet en relación con temas sanitarios son aún escasas ${ }^{14-16}$. Hasta donde sabemos, no hay publicados estudios sobre drogas con un abordaje similar al de éste, lo que hace imposible la comparación de los resultados con los de otros autores.

La gran variedad de preguntas halladas en nuestro estudio sugiere que muchos usuarios muestran interés sobre las consecuencias que su consumo tiene para su salud y buscan fórmulas para reducir los riesgos asociados. Algunos de los resultados del estudio son congruentes con los obtenidos en investigaciones previas en consumidores de éxtasis: el elevado número de preguntas acerca de la combinación de MDMA con otras drogas concuerda con el policonsumo descrito por otros autores ${ }^{16-19}$ y los efectos adversos en nuestro estudio son muy similares a los obtenidos en encuestas a usuarios de MDMA y ensayos clínicos en los que se ha administrado la droga 17,18,20-22 .

Otro tipo de cuestiones, que no se encuentran descritas en la literatura científica o sólo citadas de forma anecdótica, se repiten con una frecuencia muy elevada en los cuatro años de actividad de la lista de correo de Energy Control. De forma llamativa, al navegar en otros foros de Internet sobre MDMA (como alt.drugs.ecstasy o bluelight.nu) y páginas web sobre drogas (www.erowid.org, www.ecstasy.org, www. lycaeum.org), puede encontrarse con facilidad gran cantidad de información acerca de estos mismos temas. Este hecho sugiere que, si bien los resultados del estudio pueden no reflejar las creencias de todas las personas que han consumido MDMA en alguna ocasión, sí pueden considerarse representativos de las dudas, creencias y actitudes al menos de aquel grupo de consumidores que tiene acceso a Internet y que utiliza este medio para buscar información sobre drogas.

Uno de los temas repetitivos en la lista de Energy Control y otras fuentes de Internet son las supuestas propiedades del triptófano como fármaco que puede aliviar efectos adversos posteriores al consumo de MDMA. Se han vinculado algunos efectos adversos agudos de tipo psicológico (depresión, irritabilidad) a la deplección de los depósitos neuronales de serotonina que sigue al consumo de $\mathrm{MDMA}^{22,23}$. Al administrar un suplemento de triptófano, precursor de la biosíntesis de serotonina, teóricamente se podría incrementar la cantidad del neurotransmisor disponible, produciendo el alivio de estos síntomas. En la lista de EnergyControl la mayoría de las preguntas hacen referencia a la eficacia, seguridad y posología de esta combinación. Cada una de estas cuestiones suele ser contestada por varias personas, quienes en algunas ocasiones refieren haber utilizado el triptófano con este objetivo con resultados contradictorios. La hipótesis de los efectos beneficiosos del triptófano puede considerarse plausible desde una perspectiva estrictamente teórica y se apoya además en algunos datos anecdóticos ${ }^{24}$. Pero no existe evidencia científica sobre la eficacia y seguridad de esta combinación, acerca de la cual algunos autores han descrito posibles riesgos ${ }^{12}$. Por todos estos motivos su uso no puede recomendarse desde un punto de vista científico.

En otras ocasiones los usuarios plantean preguntas acerca de las dosis y pautas de consumo del triptófano, antioxidantes (beta-caroteno, vitamina C, vitamina $E$, ácido lipóico...) e ISRSs para protegerse de los posibles efectos neurotóxicos de la MDMA. EI consumo simultaneo de fluoxetina y MDMA con este objetivo está descrito en la literatura científica ${ }^{25}$. Todos estos fármacos han demostrado efectos neuroprotectores en el sistema nervioso central de diversas especies de animales de experimentación a los que se ha administrado $\mathrm{MDMA}^{26-29}$. Pero en este tipo de estudios se administran dosis muy elevadas tanto de MDMA como de neuroprotectores con el objetivo de estudiar en detalle los mecanismos bioquímicos responsables de la neurotoxicidad. Los resultados obtenidos en animales no son indicativos de los posibles efectos neuroprotectores en humanos, sin que sea posible hacer extrapolación alguna. En otro tipo de estudios se han administrado dosis controladas de MDMA e ISRS 30,31 a voluntarios en condiciones de laboratorio. El objeto de investigación de estos ensayos son las propiedades farmacodinámicas de la MDMA y no sus efectos neurotóxicos, sobre los que no existen métodos de medición válidos y fiables en humanos universalmente admitidos ${ }^{4,6}$. Al igual que en el caso del triptófano no es posible desde un punto de vista científico recomendar el consumo de ningún fármaco o suplemento para eliminar o atenuar los posibles efectos neurotóxicos de la MDMA.

La consulta acerca de los efectos que puede producir una pastilla determinada es la pregunta concreta más frecuentemente formulada en nuestro estudio. Este tipo de pregunta nos aproxima a ciertas creen- 
cias erróneas de algunos consumidores que pueden traducirse en comportamientos de mayor riesgo. En el mercado pueden encontrarse distintas pastillas de características externas muy variadas (color, tamaño y logo) que teóricamente facilitarían su distinción. La gran mayoría de las pastillas contienen MDMA como principio activo en distintas cantidades, generalmente entre 50 y 100 mg $^{29}$. En principio, pastillas idénticas que se encuentren en una zona geográfica determinada y en un mismo momento pueden pertenecer a un mismo lote (y por tanto tener concentraciones similares de MDMA), pero también es frecuente que coexistan distintos lotes de pastillas con ciertos logos populares. Por tanto las características externas de una pastilla no pueden utilizarse como método para obtener información acerca de su contenido.

Además algunos consumidores establecen una relación entre el aspecto externo de las pastillas y sus propiedades. Frente a los "efectos mimosistas" de la gran mayoría de los comprimidos (en referencia a las cualidades entactogénicas de la MDMA), otros producirían efectos alucinógenos ("pastillas triposas") o depresores ("pastillas jamarosas"). Los datos objetivos disponibles acerca de la composición del éxtasis en España no justifican estas creencias, ya que la adulteración o mezcla con sustancias distintas a la MDMA son anecdóticas ${ }^{32}$. Muy probablemente la explicación puede encontrarse en las diferencias en las dosis consumidas así como en las expectativas de los usuarios ${ }^{33} \mathrm{El}$ mito de que otras presentaciones del éxtasis (polvo o cristal de MDMA) producen efectos diferentes a los de las pastillas puede explicarse de forma similar.

En algunas preguntas pueden encontrarse riesgos para la salud muy importantes: la interacción farmacológica entre la MDMA y algunos fármacos como inhibidores de la monoaminooxidasa (IMAOs) ${ }^{34,35} \mathrm{o}$ el fármaco antiVIH ritonavir ${ }^{36}$ es potencialmente mortal

- El uso de otras vías de administración diferentes a la vía oral puede incrementar los efectos tóxicos de la MDMA de forma dramática. El hecho de que estas cuestiones y otras (interacción con la píldora anticonceptiva, riesgos del consumo durante la menstruación o cuando se padecen diversas enfermedades) se aborden de forma frecuente en foros de Internet debe servir como elemento de reflexión. Estudios recientes señalan que para los jóvenes norteamericanos las fuentes de información más fiables sobre la MDMA son los amigos e Internet7, valorándose mejor las páginas con información divulgativa que las páginas web oficiales de lucha antidroga7,12. En el caso concreto de la lista de correo de Energy Control, tras la revisión de los más de 15.000 mensajes la impresión general es que la participación continuada de profesionales con amplios conocimientos sobre la MDMA garantiza respuestas adecuadas en una gran mayoría de los casos.
El valor de este tipo de foros como herramienta preventiva es una cuestión particularmente interesante y no exenta de polémica. Se trata de un espacio que permite intervenir a muchas personas en tiempo real y de forma democrática, lo que facilita la transmisión de información de forma rápida y eficaz. El hecho de que todos los miembros puedan participar enriquece el debate aportando opiniones y puntos de vista muy distintos. Pero si los mensajes son excesivos, parciales, contradictorios o no se enfocan desde el ámbito de la prevención el efecto final puede ser distinto al deseado. La discusión acerca de los objetivos de los foros, población a la que debe ir dirigido, métodos, requisitos y normas, necesidad de moderadores, papel de los profesionales... son aspectos de interés, pero que exceden los límites de los objetivos de esta investigación.

\section{5.- CONCLUSIONES}

El análisis de las preguntas sobre la MDMA de la lista de correo de Energy Control aporta información valiosa acerca de las pautas de consumo, formas de uso, mitos y creencias de los consumidores sobre esta sustancia. El análisis de otros foros en Internet sobre ésta y otras drogas, así como el uso de otras metodologías de investigación (fundamentalmente la cualitativa) pueden ayudar a obtener información acerca de fenómenos cambiantes y de reciente instauración.

En ocasiones se ha presentado a Internet como un mero facilitador para el consumo de drogas. Pero el uso de canales alternativos para obtener información sobre drogas puede estar indicando una dificultad para encontrar respuestas en los profesionales sanitarios, motivada por miedo, desconfianza o bien porque la información proporcionada a través de canales tradicionales no se adecúa a las expectativas de los consumidores. La capacidad de los profesionales de la salud y de la prevención en drogas para dar respuestas concretas a colectivos concretos, de una forma objetiva y sin tabúes, determinará la evolución de este fenómeno. Internet no es más que un instrumento y como cualquier otro recurso, está sujeto a sus propias ventajas y limitaciones.

\section{AGRADECIMIENTOS}

A José Carlos Bouso Saiz, Eduardo Hidalgo Downing, Josep Rovira y Santiago Tena, miembros de Energy Control por su ayuda y aportaciones a este trabajo. 


\section{REFERENCIAS}

1. Encuesta domiciliaria sobre el consumo de drogas 2001. DGPNSD. Observatorio Español sobre Drogas. Madrid 2001.

2. Encuesta sobre drogas a la población escolar 2002. DGPNSD. Observatorio Español sobre Drogas. Madrid 2002.

3. Green AR, Mechan AO, Elliott JM, O'Shea E, Colado MI.The pharmacology and clinical pharmacology of 3,4methylenedioxymethamphetamine (MDMA, "ecstasy"). Pharmacol Rev. 2003; 55:463-508.

4. Cole JC, Sumnall HR.Altered states: the clinical effects of Ecstasy. Pharmacol Ther. 2003; 98: 35-58.

5. Reneman L.Designer drugs: how dangerous are they? J Neural Transm Suppl. 2003:61-83.

6. Kish SJ How strong is the evidence that brain serotonin neurons are damaged in human users of ecstasy? Pharmacol Biochem Behav. 2002; 71: 845-55.

7. Falck RS, Carlson RG, Wang J, Siegal HA. Sources of information about MDMA (3,4-methylenedioxymetha mphetamine): perceived accuracy, importance, and implications for prevention among young adult users. Drug and Alcohol Dependence, 2004; 73.

8. Bogenschutz MP. Drug information libraries on the Internet J Psychoactive Drugs 2000; 32: 249-58.

9. Wax PM. Just a click away: recreational drug Web sites on the Internet. Pediatrics. 2002 Jun; 109.

10. Halpern JH Pope HG. Hallucinogens on the Internet: a vast new source of underground drug information. Am J Psychiatry 2001; 158: 481-3.

11. Szalavitz MWeb sites and misinformation about illicit drugs. N Engl J Med. 2001; 345: 1710.

12. Boyer EW, Shannon M, Hibberd PL. Web sites with misinformation about illicit drugs. N Engl J Med.2001; 345:469-71.

13. Weir E. Raves: a review of the culture, the drugs and the prevention of harm CMAJ. 2000;162:1843-8.

14. Hernandez-Borges AA, Pareras LG, Jimenez A. Comparative analysis of pediatric mailing lists on the Internet. Pediatrics. 1997 Aug; 100: 82-88.

15. McLauchlan GJ, Cadogan M, Oliver CW.Assessment of an electronic mailing list for orthopaedic and trauma surgery. J R Coll Surg Edinb. 1999 Feb; 44, 36-9

16. Mursch K, Behnke-Mursch J. Internet-based interaction among brain tumour patients. Analysis of a medical mailing list. Zentralbl Neurochir. 2003; 64:71-5.

17. Solowij N, Hall W, Lee N Recreational MDMA use in Sydney: a profile of 'Ecstacy' users and their experiences with the drug, Br J Addict 1992;87:1161-72.

18. Gamella J. F. y Álvarez-Roldán, A. Drogas de síntesis en España. Patrones y tendencias de adquisición y consumo, Madrid, 1997. Ministerio del Interior,
Publicaciones de la Delegación de Gobierno para el Plan Nacional sobre Drogas. Delegación del Gobierno para el Plan Nacional sobre Drogas.

19. de la Fuente de Hoz L, Rodríguez Arenas MA, Vicente Orta J, Sánchez Paya J, Barrio Anta G. Epidemiología del consumo de drogas de diseño en España. Med Clin (Barc) 1997; 108:54-61.

20. Vollenweider FX, Gamma A, Liechti M, Huber T.Psychological and cardiovascular effects and shortterm sequelae of MDMA ("ecstasy") in MDMA-naive healthy volunteers. Neuropsychopharmacology 1998; 19:241-51.

21. Liechti ME, Vollenweider FX.Acute psychological and physiological effects of MDMA ("Ecstasy") after haloperidol pretreatment in healthy humans.Eur Neuropsychopharmacol 2000;10:289-95.

22. Curran HV, Travill RA. Mood and cognitive effects of +/-3, 4-methylenedioxymethamphetamine (MDMA, 'ecstasy'): week-end 'high' followed by mid-week low. Addiction. 1997; 92:821-31.

23. McKenna DJ, Peroutka SJ. Neurochemistry and neurotoxicity of 3, 4-methylenedioxymethamphetamin e (MDMA, "ecstasy"). J Neurochem. 1990; 54:14-22.

24. Schifano F, Magni G.MDMA ("ecstasy") abuse: psychopathological features and craving for chocolate: a case series. Biol Psychiatry 1994;36:763-7.

25. McCann UD, Ricaurte GA.Reinforcing subjective effects of (+/-) 3,4-methylenedioxymethamphetami ne ("ecstasy") may be separable from its neurotoxic actions: clinical evidence.J Clin Psychopharmacol. 1993;13: 214-7.

26. Sanchez V, Camarero J, Esteban B, Peter MJ, Green AR, Colado MI.The mechanisms involved in the long-lasting neuroprotective effect of fluoxetine against MDMA ('ecstasy')-induced degeneration of 5-HT nerve endings in rat brain. Br J Pharmacol. 2001;134: 46-57.

27. Aguirre N, Barrionuevo M, Ramirez MJ, Del Rio J, Lasheras B.Alpha-lipoic acid prevents 3,4methylenedioxy-methamphetamine (MDMA)-induced neurotoxicity. Neuroreport. 1999;10: 3675-80.

28. Shankaran M, Yamamoto BK, Gudelsky GA.Ascorbic acid prevents 3,4-methylenedioxymethamphetamine (MDMA)-induced hydroxyl radical formation and the behavioral and neurochemical consequences of the depletion of brain 5-HT. Synapse. 2001;40: 55-64.

29. Sprague JE, Huang $X$, Kanthasamy A, Nichols DE. Attenuation of 3,4-methylenedioxymethamphetami ne (MDMA) induced neurotoxicity with the serotonin precursors tryptophan and 5-hydroxytryptophan. Life Sci. 1994;15: 1193-8.

30. Pacifici R, Pichini $S$, Zuccaro P, Farre $M$, Segura $M$, Ortuno J, Di Carlo S, Bacosi A, Roset P, Segura J, De La Torre R. Paroxetine Inhibits Acute Effects of MDMA 
on the Immune System in Humans. J Pharmacol Exp Ther. 2004 Jan 13.

31. Liechti ME, Vollenweider FX.The serotonin uptake inhibitor citalopram reduces acute cardiovascular and vegetative effects of 3,4-methylenedioxymet hamphetamine ('Ecstasy') in healthy volunteers.J Psychopharmacol. 2000;14: 269.

32. Memoria 2001. Sección de Drogas de Madrid. Instituto Nacional de Toxicología.Madrid, 2001.

33. McElrath K, McEvoy K. Negative experiences on Ecstasy: the role of drug, set and setting. J Psychoactive Drugs. 2002; 34:199-208.
34. Smilkstein MJ, Smolinske SC, Rumack BH. A case of MAO inhibitor/MDMA interaction: agony after ecstasy. J Toxicol Clin Toxicol. 1987; 25:149-59.

35. Vuori E, Henry JA, Ojanpera I, Nieminen R, Savolainen T, Wahlsten $P$ et al.Death following ingestion of MDMA (ecstasy) and moclobemide. Addiction. 2003; 98:365-8.

36. Antoniou T, Tseng AL. Interactions between recreational drugs and antiretroviral agents. Ann Pharmacother. 2002; 36:1598-613. 